\title{
SIMULATION OF ARMY UNMANNED AERIAL VEHICLE COMMUNICATIONS
}

\author{
Richard Chapman \\ Drew Hamilton \\ Daniel Box \\ Mark Kuhr \\ Jonathan MacDonald \\ Stephen Hamilton \\ Computer Science and Software Engineering \\ 107 Dunstan Hall, Auburn University \\ Auburn, AL 36849, USA
}

\begin{abstract}
We explore development of a high-fidelity simulation testbed for various network architectures for communication between components of tactical unmanned aerial systems, and for distribution of intelligence gathered by these systems.
\end{abstract}

\section{INTRODUCTION}

Unmanned aerial systems (UAS's) have proven very successful at gathering tactical intelligence. Systems such as the army RQ-11 Raven and RQ-7 Shadow have provided commanders on the ground with much valuable information (Dickerson 2007). The current generation of tactical UAS's typically operate as a single air vehicle controlled by single ground station, or perhaps as a group of several independent air vehicles sharing a common ground station, rather than as participants in a digital network.

The joint Future Combat System (FCS) program aims for a networked battlespace, in which all participants can communicate on a common network. Various initiatives support this vision, such as the Unmanned Systems Initiative which funded our initial work. As noted by Senator Richard Shelby (R-AL) (Shelby 2005), "The Unmanned Systems Initiative (USI) will conduct research and development of unmanned systems to advance, evaluate, and rapidly mature technologies to meet urgent warfighter needs as well as the optimal tactics, techniques and procedures to employ unmanned systems capabilities.

Unmanned systems have immense potential to reduce engagement timelines while improving survivability in difficult urban terrain. Acceleration of the transition and fielding of these robotic technologies and capabilities is a critical wartime effort."
The ground system of a typical UAS consists of a control station or operating position consisting of several computers and a user interface for the human operator, a launcher, an uplink radio transceiver and antenna, and perhaps a radar of some sort to assist in landing. Cabling between these components can weigh hundreds of pounds, require significant time to set up and take down (a particular concern in areas of insurgent activity, where there is concept of a safe 'rear area' from which to conduct UAV operations), and limit flexibility in the configuration or use of the UAS. Additionally, army tactical unmanned aerial systems communication architectures today typically route all communication with the air vehicle through a control station which is also responsible for controlling and monitoring the vehicle.

However, the payload information, such as video from a camera aimed at the ground is generally desired by some other entity, commonly a tactical unit near the air vehicle's position. Thus, the payload information must be somehow routed 'forward' from the control station to the tactical unit, or to multiple tactical units. These units may be physically much closer to the air vehicle itself, which is gathering information relevant to the unit's current mission, than to the control station (see Figure 1). The payload data may be routed by a variety of different networks, or a combination of networks, in order to deliver it to the tactical unit in a timely manner. Our simulation is a first step in the exploration of delivering the information directly from the air vehicle to the interested tactical units by a wireless '802.11-type' network, or a combination of wired and wireless battlefield TCP/IP networks.

Once the air vehicle, ground station, and other UAS components are participating in a TCP/IP network, other sorts of information sharing become possible, including the sharing of information needed for formation flying by a group of vehicles, or for close cooperation with manned 
aircraft or ground vehicles, as well as unmanned ground vehicles. One particularly appealing application would be the use of a UAV as a 'spotter' for an unmanned ground vehicle planning its motion over unknown or hostile terrain.

\section{SIMULATION OF GROUND SYSTEM COMPONENT COMMUNICATION}

The objective of the simulation of ground system component communication was the facilitation of replacement of cabling with a wireless network. The simulation was calibrated using data gathered from real point-to-point TCP/IP links carrying UAS traffic between ground system components. The links used 802.11a with additional layers of encryption provided by Cisco 5510 security appliances. Testing was done at various combinations of distance, antenna type, protocol, and power level, using network adapters and access points from a variety of manufacturers. Data gathered included latency, dropped packets, and quality measures for real-time streaming video.

Traffic data from the field tests was gathered using Wireshark, and traffic flows in the simulator (see section 4) were generated based on these tests. Data gathered in this phase can also be used for simulation of various dissemination architectures (see section 3).

\section{SIMULATION OF DATA DISSEMINATION ARCHITECTURES}

Army tactical unmanned aerial vehicles currently communicate predominantly by a dedicated radio modem with a single ground control station. Wider dissemination of payload information is handled in a variety of ways, but in nearly all cases the information must pass through the choke point of the ground control station before being routed to the consumers of the payload information ( see Figure 1).

One alternative would be for the air vehicle to broadcast or multicast payload information to any authorized tactical units in its vicinity directly (see Figure 2). This has the advantage of simplicity - the network architecture is simply that of a wireless LAN. Depending on the frequency band used, participants in the network might require a clear line of sight to the air vehicle. However, terrain and vegetation blocking line of sight communications between the units on the ground would not impair communication of the air vehicle payload to all ground units.

A second alternative would be for one, or a small number of ground units to communicate directly with the air vehicle. The majority of ground units would not communicate directly with the air vehicle, but would receive the information via a terrestrial wireless network in which the units that have received the payload broadcast forward it to other interested units, perhaps in the manner of peerto-peer ( $22 p)$ file streaming systems on the Internet today.

The goal of our simulation is to compare the three scenarios described above in terms to determine the relationship between power levels, antenna gain, and maximum separation of communicating units in realistic simulation tactical situations. Particular factors to be simulated include

- Curvature of the earth

- Radio propagation in the atmosphere

- Atmospheric moisture

- Effects of terrain

- Effects of buildings and vehicles

- Effects of foliage

\section{SIMULATORS}

OPNET, Qualnet, and ns2 were considered for simulation of the three communications architectures outlined above. Ns2 is an open-source discrete even simulator widely used for networking research. It provides substantial support for simulation of TCP, routing, and multicast protocols over wired and wireless (local and satellite) networks (Information Science Institute 2006). Qualnet is a commercial product of Scalable Network Technologies (Qualnet 2007) (see Figure 5). It possesses the features of ns2 mentioned above, as well as the ability to simulate IPSec and WiMax out of the box. The Qualnet toolset also include a GUI. OPNET is a similar program (OPNET 2007).

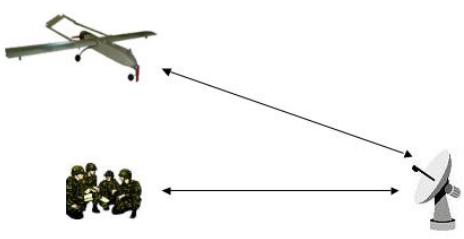

Figure 1: Current communication architecture

While the open-source nature of ns 2 makes it possible to implement just about any feature desired, the tradeoff is that the implementation takes time. In particular, the ease with which Qualnet enables modeling a system in which different nodes transmit with different power levels, and in which different nodes may use antennas with different gains and different directional characteristics, was a 
strong point in its favor, and is consequently the simulator we are currently using.

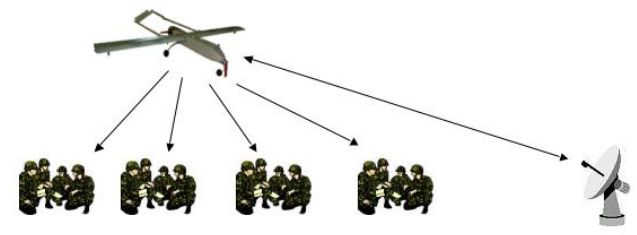

Figure 2: Dissemination to multiple recipients via broadcast

\section{BASE MODEL}

Initially we consider transmission from one UAV to a single ground station. For the ground station we used the OSRVT ROVER II, a simplex, point-to-point, Line-ofsight, multi-band communications data link for in imagery and telemetry collection systems (L-3 Communications 2006). It is able to receive L-band (390Mhz to $1.55 \mathrm{Ghz}$ ), C-band (3-7Ghz), and Ku-band (11-15Ghz) transmissions. The C-band was used for the base model. Both receive and transmit antennas were omnidirectional. The receiver model consists of antenna, LNA, and RF filter, operating on the frequencies of 4.40 to $5.85 \mathrm{Ghz}$.

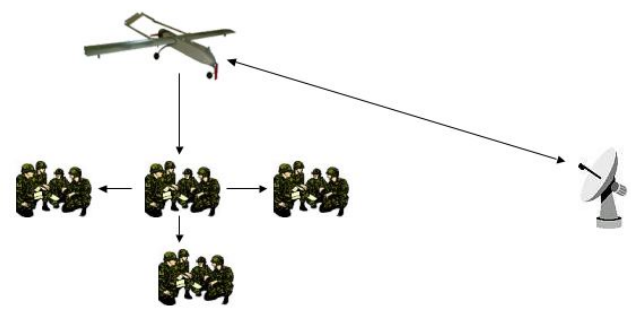

Figure 3: Dissemination to a hub unit followed by terrestrial dissemination

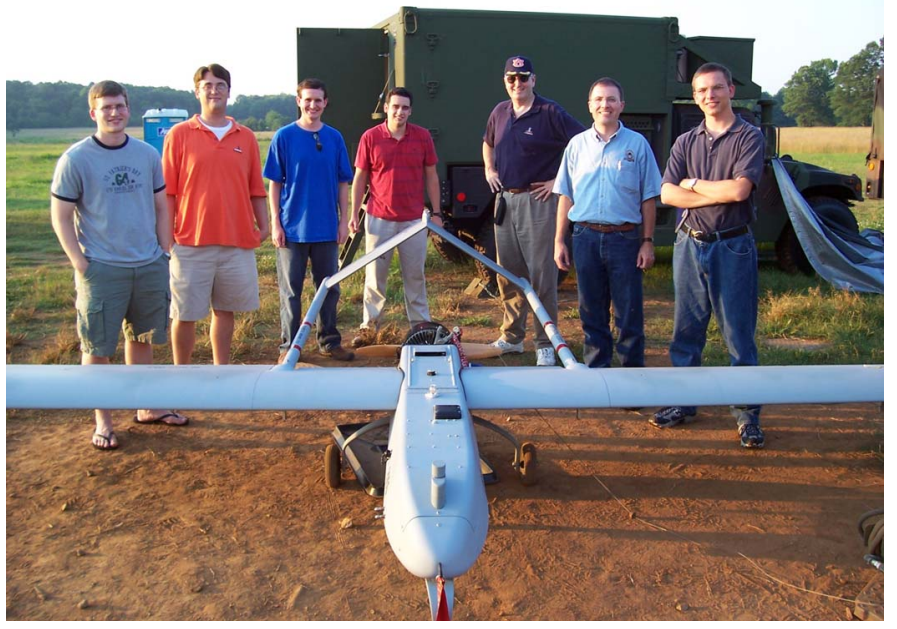

Figure 4: Auburn UAV research team with UAV from the 101st Airborne Division at Redstone Arsenal. From left to right: Alan Hunt, Kevin Richardson, Shawn Constance, Daniel Box, Drew Hamilton, David Umphress and David Last

\begin{tabular}{|c|c|c|c|c|c|c|}
\hline $\begin{array}{l}108.419 \\
m\end{array}$ & $\begin{array}{l}2833.100 \\
11\end{array}$ & $\begin{array}{l}359.797 \\
m\end{array}$ & \begin{tabular}{|l}
496,496 \\
m
\end{tabular} & $\begin{array}{l}613.176 \\
m\end{array}$ & $\begin{array}{l}7392055 \\
m\end{array}$ & $\begin{array}{l}200 \mathrm{E} E \\
\mathrm{mi}\end{array}$ \\
\hline
\end{tabular}

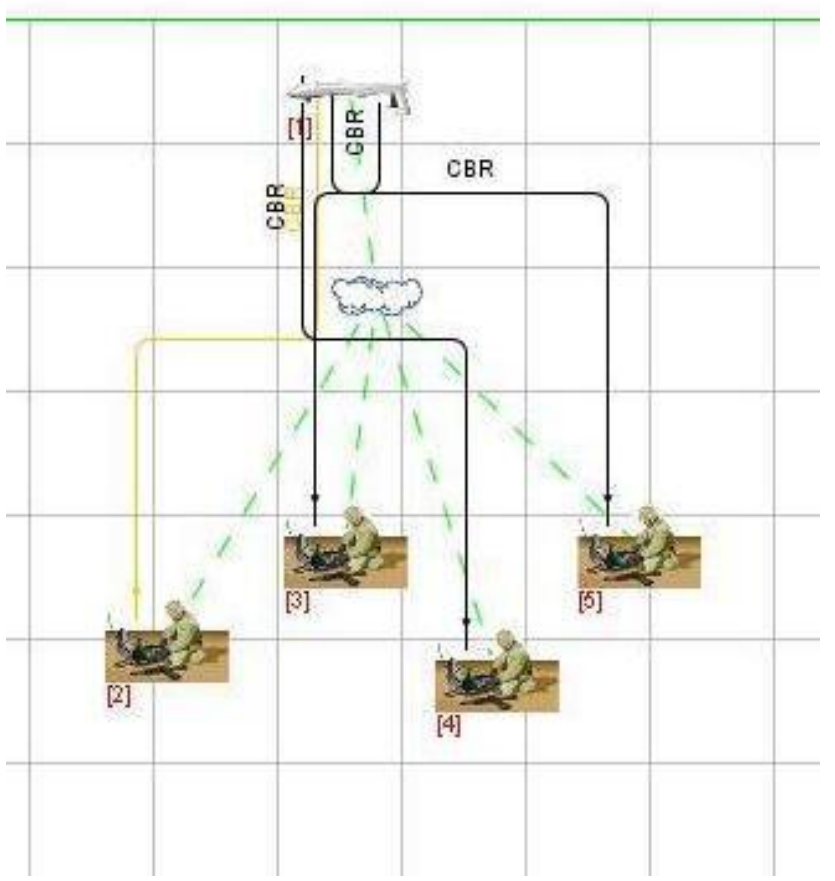

Figure 5: Qualnet simulation: A UAV sends a video stream to four stations on the ground. 


\section{FUTURE WORK}

The base model must first be extended to handle the obstacles to transmission outlined in section 3. Next, scenarios will be developed to model the two dissemination architectures, followed by verification and validation of the simulation.

\section{CONCLUSION}

The simulation framework described is just the initial step toward developing a framework for high-fidelity simulation of unmanned aerial systems communications. As such systems are deployed more widely in military applications, and eventually in civilian applications, simulation will be an important tool in enhancing the functionality of the systems through networking.

\section{ACKNOWLEDGEMENTS}

The researchers acknowledge the support of the Unmanned Systems Initiative, administered by the Joint Technology Center/System Integration Laboratory at Redstone Arsenal, Alabama, and the Army UAS Program Office, also at Redstone Arsenal.

\section{REFERENCES}

Dickerson, L. 2007. UAV's on the Rise. Aviation Week and Space Technology, January 15, 2007.

Shelby, Senator R. C. 2005. Press Release: Shelby Announces Defense Funding for North Alabama. 7 Oct 2005, <shelby. senate.gov/news/record. cfm? id=247067> [accessed 20 June 2007]

Information Science Institute. 2006. The Network Simulator - NS2. April 2006. Available at: <www.isi.edu/nsnam/ns/>

Scalable Network Technologies. 2007. <qualnet.com> [Accessed August 1, 2007].

OPNET Technologies, 2007 <www. opnet.com> [Accessed August 1, 2007].

L-3 Communications. 2006. Prime Item Development Specifications for the OSRT Rover III. March 28, 2006.

\section{AUTHOR BIOGRAPHIES}

RICHARD CHAPMAN is an associate professor in Auburn University's Department of Computer Science and Software Engineering, and director of the Center for Innovation in Mobile, Pervasive, Agile, Computing Technology (iMPACT). His web page can be found via <www. eng • auburn. edu/ chapman>
DREW HAMILTON is an associate professor in Auburn University's Department of Computer Science and Software Engineering, and director of the Information Assurance Center at Auburn. His web page can be found via <www.eng. auburn. edu/ hamilton>

DANIEL BOX, MARK KUHR, STEPHEN HAMILTON, AND JONATHAN MACDONALD are graduate students in Auburn University Department of Computer Science and Software Engineering, specializing in computer simulation and information assurance. 